\title{
Two-month consumption of bread enriched with a fiber mix: impact on gut microbiota and cardiometabolic profile in at cardiometabolic-risk subjects.
}

\author{
Harimalala Ranaivo ${ }^{1}$, Susie Guilly ${ }^{2}$, Monique Sothier ${ }^{1}$, Laurie Van Den Berghe ${ }^{1}$, \\ Stéphanie Lambert-Porcheron ${ }^{1}$, Isabelle Dussous, Loïc Roger, Christel Béra-Maillet ${ }^{4}$, \\ Hugo Roume ${ }^{2}$, Nathalie Galleron ${ }^{2}$, Nicolas Pons', Emmanuelle Le Chatelier ${ }^{2}$, Dusko Ehrlich ${ }^{2}$, \\ Martine Laville ${ }^{1}$, Joël Doré ${ }^{2}$ and Julie-Anne Nazare ${ }^{1}$ \\ ${ }^{1}$ Centre de Recherche en Nutrition Humaine Rhône-Alpes, Univ-Lyon, CarMeN Laboratory, Université Claude Bernard \\ Lyon1, Hospices Civils de Lyon, CENS, FCRINIFORCE Network, Pierre-Bénite, France, \\ ${ }^{2}$ INRA, Metagenopolis, Jouy-en-Josas, France, \\ 3Bridor, Rennes, France and \\ ${ }^{4}$ INRA, Micalis, Jouy-en-Josas, France
}

\begin{abstract}
Introduction

Increased adiposity, dyslipidemia and insulin resistance are associated with increased risk of developing cardiometabolic diseases (CM). Such deleterious phenotypes have been shown to be associated with a low gene-richness microbiota that can partly be restored by a short-term dietary intervention (energy-restricted high-protein diet, low glycemic index, enrichment with fibers) in parallel to an improvement of CM profile. In this study, we aimed at increasing fiber intake in quantity and diversity through a two-month consumption of bread enriched with a mix of selected fibers and evaluated the impact of this dietary intervention on gut microbiota gene richness and $\mathrm{CM}$ risk profile in subjects at risk of developing CM.
\end{abstract}

\section{Materials and methods}

In a randomized double blind cross-over design, thirty-nine subjects with CM risk profile (18-70 years old, BMI: $25-35 \mathrm{~kg} / \mathrm{m}^{2}$, waist circumference $>80 \mathrm{~cm}$ for women and $>96 \mathrm{~cm}$ for men, fiber intake $<20 \mathrm{~g} / \mathrm{day}$, low fiber diversity) consumed daily for $8 \mathrm{weeks} 150 \mathrm{~g}$ of standard bread vs. $150 \mathrm{~g}$ of bread enriched with a 7-selected fibers mix ( $5.55 \mathrm{~g}$ vs. $16.35 \mathrm{~g}$ of fiber respectively; 4-week washout). Gut microbiota and CM risk factors' analyzes were conducted before and after intervention. Stool samples were analyzed by shotgun metagenomics; microbial genes and metagenomics species (MSP) profiles were generated by mapping reads on a reference genes cata$\log (1529 \mathrm{MSP})$.

\section{Results}

The included dyslipidemic subjects with CM risk profile presented a lower microbiota gene richness compared to reference healthy cohorts. The two-month consumption of fiber-rich bread did not alter microbiota gene richness but modified microbiota composition with a significant decrease of Bacteroides vulgatus $(\mathrm{q}=1.7 \mathrm{e}-4)$ and a significant increase of Parabacteroides distasonis $(\mathrm{q}=2.8 \mathrm{e}-6)$, Fusicatenibacter saccharivorans $(\mathrm{q}=5 \mathrm{e}-5)$ and Clostridiales $(\mathrm{q}=3.8 \mathrm{e}-2)$. We observed in parallel a significant decrease in total cholesterol $(-0.26 \mathrm{mmol} / \mathrm{L} ;-5 \% ; \mathrm{p}=0.021)$, LDL-cholesterol $(-0.2 \mathrm{mmol} / \mathrm{L} ;-6 \%, \mathrm{p}=0.0061)$ and an improvement of insulin sensibility estimated by HOMA index (3.23-2.54 mUI/L; - 21\%; $\mathrm{p}=0.0079)$. These effects were even significantly more pronounced for subjects presenting the higher waist circumference. Anthropometric parameters were not altered.

\section{Discussion}

The enrichment of the diet with a mix of selected fibers for 2 months altered microbiota composition by modifying the relative abundance of specific gut bacterial species, in parallel to a significant improvement of cholesterol and insulin sensitivity parameters. Increasing the quantity and diversity of dietary fiber intake could be used as an efficient tool to favorably impact CM profile.

\section{Conflict of Interest}

This study was funded by Bridor. I.Dussous and L.Roger work for Bridor as an employee and consultant respectively. The remaining authors declare no conflict of interest. 